\title{
VIOLENCIAS ESTRUCTURALES: OBSTÁCULOS PARA EL CUMPLIMIENTO DE LOS DERECHOS HUMANOS DE LAS MUJERES POBRES
}

Ma ASUNCIÓN MARTÍNEZ ROMÁN

Universidad de Alicante

\section{LAS VIOLENCIAS ESTRUCTURALES CONTRA LA MUJER SON VIOLACIONES DE SUS DERECHOS HUMANOS}

En el siglo XXI ser mujer todavía implica un alto riesgo de sufrir discriminación y desigualdad de oportunidades, en comparación a las que disfrutan los hombres, sin ninguna justificación o razón para ello más que el peso de la violencia contra las mujeres ejercida por la cultura y la estructura social, económica y política durante siglos, por lo que estamos ante una evidente injusticia social. Revertir esta situación y garantizar la no-discriminación y la igualdad de oportunidades entre mujeres y hombres es una cuestión de derechos humanos.

Esto implica, en primer lugar, el reconocimiento social de la existencia de los obstáculos que impiden el pleno ejercicio de los derechos humanos reconocidos a toda persona humana $y$, además, adoptar las medidas de toda índole necesarias para eliminar los obstáculos, verificando el cumplimiento de dichas medidas. Naciones Unidas promueve el cambio a nivel mundial desde la Convención sobre la eliminación de todas las formas de discriminación contra la mujer ${ }^{1}$, en cuyo Art. 1 se define la existencia de la discriminación contra la mujer como

«Toda distinción, exclusión a restricción basada en el sexo que tenga por objeto o por resultado menoscabar o anular el reconocimiento, goce o ejercicio por la mujer, independientemente de su estado civil, sobre la base de la igualdad del hombre y la mujer, de los derechos humanos y las libertades fundamentales en las esferas política, económica, social, cultural y civil o en cualquier otra esfera».

Sin embargo, hay muchas resistencias al cambio propuesto y está siendo difícil modificar las causas que generan estas discriminaciones basadas en cons-

1. Aprobada el 18 de diciembre de 1979 por la Asamblea General de las Naciones Unidas, entró en vigor como tratado internacional el 3 de septiembre de 1981 tras su ratificación por 20 países. 
trucciones sociales de los roles de mujeres y hombres, que han venido asignado tradicionalmente al hombre el poder de decisión de forma manifiestamente desigual respecto a la mujer. En consecuencia, se han establecido y reforzado a través de los siglos unas relaciones desiguales en las que el hombre ha llegado a considerar natural ejercer el poder, controlando los recursos y tomando decisiones sin contar con la mujer e, incluso, logrando que la mujer llegara también a considerarlo natural.

Enmarcado en el tema de los derechos humanos, en la Declaración de Viena se vuelve a insistir en la necesidad de promover la igualdad de oportunidades para la mujer recuperando el poder de decisión sobre su propia vida:

"Los derechos humanos de la mujer y de las niñas son parte inalienable, integral e indivisible de los derechos humanos universales. La total e igual participación de la mujer en la vida política, civil, económica, social y cultural en los niveles nacional, regional e internacional y la erradicación de todas las formas de discriminación por razón de sexo son objetivos prioritarios de la comunidad internacional»².

La necesidad de incluir la ratificación del reconocimiento de los derechos humanos para la mujer y las niñas indica el reconocimiento de la envergadura de las discriminaciones sufridas por la mujer en todo el mundo, con mayor gravedad en los países en desarrollo, pero también en los países desarrollados. En 1995, los acuerdos firmados por los gobiernos participantes en la Cuarta Conferencia Mundial sobre la Mujer plasmados en la Declaración y la Plataforma de Acción $^{3}$ incorporan lo logrado en todas las Conferencias y Tratados anteriores ${ }^{4}$. El cumplimiento de las actuaciones previstas en la Plataforma de Acción de Beijing es objeto de un seguimiento que da lugar a Informes de evaluación como el realizado en el año $2000^{5}$, constatando los avances conseguidos en cuanto a los derechos de la mujer en el marco de los derechos humanos (con desiguales resultados entre países y en el interior de los países), subrayando la necesidad de continuar integrando una perspectiva de género en la elaboración de políticas y programas y reafirmando el convencimiento de que una igualdad de géneros beneficiaría no solo a las mujeres, sino a la sociedad en su conjunto 6 . A partir de 1995 ya no valen las excusas. Es cuestión de voluntad política porque resulta

2. NACIONES UNIDAS: Conferencia sobre Derechos Humanos, Viena 1993. Declaración y Programa de Acción, Parte I, 18.

3. NACIONES UNIDAS: Informe de la Cuarta Conferencia Mundial sobre la Mujer, Beijing, 4-15 de septiembre de 1995, cap I, resolución 1, anexos I y II.

4. Declaración Universal de los Derechos Humanos; Declaración de la Eliminación de la Discriminación contra la Mujer; la Declaración de Viena sobre Derechos Humanos (1993); la Conferencia de Población y Desarrollo de El Cairo (1994).

5. NACIONES UNIDAS: La Mujer en el año 2000: igualdad entre los géneros, desarrollo y paz para el siglo XXI. Nueva York, 5-9 de junio de 2000. Periodo extraordinario de sesiones de la Asamblea General de Naciones Unidas.

6. La última revisión de los objetivos de Beijing se ha realizado en Nueva York en marzo de 2005. NACIONES UNIDAS. Comisión de la Condición Jurídica y Social de la Mujer (CSW): Revisión y evaluación de los 10 años de la Plataforma de Acción de Beijing (Beijing +10), Nueva York, 28 de febrero a 11 de marzo. 
Violencias estructurales: obstáculos para el cumplimiento de los derechos humanos...

injustificable que, estando claramente reconocida la existencia del problema y bien definidas las medidas a adoptar, todavía persista la discriminación por razón de género, incluyendo la más visible que es la violencia personal directa ${ }^{7}$.

A pesar de haber logrado progresos indudables, éstos no son homogéneos existiendo diferencias entre países y, mayoritariamente, estamos lejos de ese necesario reconocimiento social de la discriminación por razón de género en el disfrute de los derechos humanos. De todos modos, el aumento de la sensibilización social parece estar entre las causas que han llevado a un mayor reconocimiento de la violencia personal directa contra las mujeres que no es sino la parte más visible, la punta de un iceberg formado por otras violencias como la violencia estructural y la violencia cultural, por las que se relega a las mujeres a posiciones sociales desde las que no pueden tener el control sobre sus propias vidas. El género se modela por determinantes ideológicos, históricos, religiosos, étnicos, económicos y culturales y las relaciones de género afectan a la manera en que hombres y mujeres participan en los procesos económicos, sociales y políticos que condicionan su vida.

Se suele hablar de violencia en referencia a situaciones de violencia directa a personas, por ejemplo, en conflictos bélicos. Y se habla de violencia contra la mujer en un sentido restringido, reducido a la violencia personal directa en el ámbito doméstico, lo que ha servido de excusa durante tiempo para darle la consideración social de ámbito privado sobre el que no se podía intervenir. También existen violencias indirectas menos visibles aparentemente que, además, pueden ser origen de otras violencias directas y que afectan a millones de mujeres y niñas en todo el mundo y que Galtung califica como violencia estructural. El autor sostiene una concepción amplia de la violencia, más allá de la mera violencia física, en la que se relacionan directamente paz y desarrollo considerando que «la violencia está presente cuando los seres humanos se ven influidos de tal manera que sus realizaciones efectivas, somáticas y mentales, están por debajo de sus realizaciones potenciales», de modo que "cuando lo potencial es mayor que lo efectivo, y ello es evitable, existe violencia» ${ }^{8}$. Las personas que carecen de poder de decisión sobre la distribución de los recursos existentes no tienen las mismas oportunidades de acceso a los medios precisos para desarrollar sus potencialidades personales. Esta diferencia de oportunidades origina una permanente situación de desigualdad que puede calificarse como violencia estructural o injusticia social, violencia que, a su vez, puede ser manifiesta o latente .

7. Naciones Unidas está realizando un estudio sobre todas las formas de violencia contra la mujer, sus formas, causas, consecuencias, costos sociales económicos y de salud, identificando ejemplos de las mejores prácticas. NACIONES UNIDAS: Resolución aprobada por la Asamblea General "Estudio a fondo sobre todas las formas de violencia contra la mujer». Quincuagésimo octavo período de sesiones. A/RES/58/185.

8. GALTUNG, J.: Investigaciones teóricas. Sociedad y Cultura contemporáneas, Madrid, Tecnos-Instituto de Cultura «Juan Gil-Albert», 1995, pp. 314-315.

9. Ibid, p. 320. 
Este enfoque de la violencia estructural es aplicable al caso de la discriminación de la mujer en cuanto es una forma de violencia indirecta, menos visible, que se origina cuando las estructuras sociales, económicas, culturales y políticas favorecen unas relaciones sociales basadas en jerarquías de poder, contribuyendo a mantener y reforzar las discriminaciones por razón de género o por clase social, edad, orientación sexual o raza-etnia, etc. Se ejerce violencia cuando se justifica la superioridad de unas personas con la asignación de posiciones de inferioridad a otras, lo que permite o promueve relaciones de opresión desde quienes están situados en las posiciones superiores contra quienes ocupan las posiciones inferiores, que en relación al género suelen corresponder a la mujer. Hay que insistir en que las discriminaciones por razón de género, son violaciones de los derechos humanos y una injusticia social por lo que hay que incidir sobre las estructuras que las promueven y justifican.

\section{POBREZA Y EXCLUSIÓN SOCIAL DE LAS MUJERES COMO FORMAS DE VIOLENCIA ESTRUCTURAL}

La pobreza, según Galtung, está implantada en la estructura y justificada por la cultura. Es una forma de violencia estructural o indirecta, situación en la que la violencia se genera y se manifiesta como un poder desigual y, consiguientemente, como oportunidades de vida distintas. La pobreza se apoya en la violencia cultural, es decir, la religión, la ideología, la lengua, el arte, la ciencia o la cultura profunda pueden servir para justificar y legitimar la existencia de la pobreza, considerándola algo inevitable ${ }^{10}$.

El hecho de que la pobreza y la exclusión social afecten más a las mujeres que a los hombres es otra manifestación de la violencia estructural. La situación de discriminación que afecta al género femenino se agrava aún más en el caso de las mujeres en situación de pobreza humana y exclusión social o en riesgo de estarlo, que pueden llegar a sufrir las consecuencias de una acumulación de discriminaciones y desventajas de diversa índole. Al riesgo de discriminación por razón de género hay que sumar el riesgo de discriminación por razón de edad, discapacidades o enfermedades crónicas graves, migraciones, pertenencia a una minoría cultural o religiosa, etc. La posición social de la mujer, el status marital, o la pertenencia a determinados grupos más vulnerables como mujeres refugiadas o migrantes, tener residencia en campo o ciudad, son factores que se acumulan en la discriminación ${ }^{11}$.

Una de las manifestaciones de la violencia estructural contra la mujer ha sido el ignorar la perspectiva de género en las metodologías de investigación social y económica, siendo reciente la incorporación de esta perspectiva que resulta imprescindible para poder discernir las diferencias por razón de género, tanto

10. Galtung, J.: Peace by peaceful means. Peace and Conflict, Development and Civilization, Londres, Sage-Prio, 1996.

11. ONU: Women's Rights are Human Rights. Special Issue on Women's Rights-Spring 2000. 
Violencias estructurales: obstáculos para el cumplimiento de los derechos humanos...

en los procesos de empobrecimiento como en su superación ${ }^{12}$. La falta de indicadores desde esta perspectiva ha hecho invisibles las condiciones de vida de las mujeres pobres al considerarla como un miembro más del hogar, como si las relaciones en el interior del hogar fueran igualitarias. Por ejemplo, para conocer las diferencias de acceso y disfrute de las opciones de desarrollo humano por razón de género, hay que analizar tanto la situación del hogar como la de la comunidad o el contexto social más amplio. Entre las cuestiones clave a tener en cuenta podemos señalar, en el interior del hogar; el modo de distribución de los recursos de la familia entre sus miembros, el modo de toma de decisiones, la asignación de las funciones de cuidado de los miembros dependientes, el acceso al mercado laboral, sin olvidar la distribución del tiempo personal que condiciona en gran medida la posibilidad o imposibilidad de participar activamente en la vida social y política. En cuanto al exterior del hogar, hay que analizar las estructuras de desigualdad: características del contexto sociocultural, económico y político; las oportunidades de acceso a los servicios de educación y formación profesional, salud, servicios sociales, vivienda o acceso a un empleo; responsabilidades sociales asignadas en función del género; oportunidades de apoyo social informal; oportunidades de participación en la vida social y política, etc. Además, habrá que analizar el contexto regional, nacional e internacional, factores todos ellos que no resultan ajenos a las existencia de desigualdades por razón de género ${ }^{13}$.

Otra dificultad para conocer las desigualdades por razón de género es que, a menudo, si se habla de discriminación por razón de género, se hace centrándose en la etapa adulta por cuanto interesa la aportación de las mujeres en la dimensión económicamente productiva o en su dimensión de prestadora de cuidados al núcleo familiar o ante el incremento de muertes violentas de mujeres causadas por hombres. Sin embargo, al decidir actuaciones públicas no siempre se tiene en cuenta, por ejemplo, que cuando hay obstáculos que impiden a las mujeres el acceso al mundo laboral no basta con diseñar políticas dirigidas a las mujeres en edad activa porque las discriminaciones laborales tienen también causas educativas y culturales que se originan en la infancia. Que cuando se pretende promover un reparto más igualitario de las responsabilidades familiares, no basta con adoptar algunas medidas dirigidas a las mujeres ocupadas y beneficiarias del sistema de protección social porque se está excluyendo a muchas mujeres que no tienen derecho a esa protección. Y que, cuando se diseñan políticas contra la violencia a las mujeres, no basta con actuaciones puntuales de asistencia a las víctimas de la violencia directa, aun cuando todo ello sea muy necesario.

12. MATEO, M.A.: «¿Conoces a alguien más pobre que yo? Definiciones de la pobreza desde la perspectiva de género", en Tortosa, J.M. (coord.): Mujeres pobres, indicadores de empobrecimiento en la España de hoy, Madrid, Fundación FOESSA, 2002, pp. 55-69.

13. MARTínez ROMÁN, Ma A.: "Género, Pobreza y Exclusión social: Diferentes conceptualizaciones y políticas públicas", en Tortosa, J.M. (coord.): Pobreza y Perspectiva de Género, Barcelona, Icaria, 2001, pp. 65-83. 
Resumiendo, además de reconocer las violencias visibles y no visibles contra las mujeres, hay que preguntarse a qué se deben, para actuar sobre las causas estructurales y culturales teniendo en cuenta que, la discriminación por razón de género, comienza en la infancia y se acumula a lo largo del ciclo vital de las mujeres: mujeres-niñas, mujeres-adolescentes, mujeres-adultas, mujeres- mayores y mujeres-ancianas. Por lo tanto, las políticas públicas dirigidas a prevenir y erradicar la violencia por razón de género precisan estrategias a corto, medio y largo plazo, con actuaciones globales que promuevan y garanticen el ejercicio del derecho de las mujeres a la igualdad de opciones, en comparación con los hombres, para su propio desarrollo personal en todos los ámbitos como la salud, educación, empleo, ingresos, vivienda, cultura y ocio, etc.

\section{POBREZA Y EXCLUSIÓN SOCIAL DE LAS MUJERES: EL ESTADO DE LA CUESTIÓN}

Del análisis de la situación actual se concluye que, a pesar de las actuaciones y logros a nivel mundial, el número de mujeres en situación de pobreza no solo no ha disminuido sino que continua aumentando. La falta de metodologías de medición de la pobreza con perspectiva de género y comparables sigue siendo una tarea pendiente entre las acordadas en Beijing, debido a obstáculos relacionados con brechas técnicas e institucionales, metodologías de investigación y clasificación de datos, difusión y presentación de datos y recursos financieros y humanos. Otra tarea pendiente, entre muchas, es el análisis de género de las políticas económicas y programas para promover una distribución de los recursos, la producción, la riqueza, las oportunidades, los ingresos y los servicios en forma más equitativa ${ }^{14}$. Hay que invertir urgentemente en los niños del mundo que se ven impedidos para ejercer sus derechos más básicos ${ }^{15}$. Por ejemplo, en el ámbito de la educación, se ha logrado mejorar la escolarización primaria de las niñas, aunque con diferencias regionales y entre países, pero sigue siendo muy importante para prevenir la pobreza de las niñas su acceso a la educación secundaria ${ }^{16}$. El nivel de educación de la madre desempeña una importante función para determinar si un niño asiste o no a la escuela, lo que da idea de la importancia de que las niñas asistan a la escuela y no abandonen antes de finalizar. En los países en desarrollo, el 75\% de los niños y niñas que no acuden a la escuela primaria son hijos de mujeres que no han recibido educación ${ }^{17}$. En cuanto a la educación secundaria, tan solo un 39\% de los niños y niñas en edad

14. Beijing a los 10 años: de la Política a la Práctica. Revisión y valoración de la implementación de la Declaración y Plataforma de Beijing. Área crítica A. La mujer y la pobreza. 2004 www.un-instraw.org

15. UNICEF: Estado Mundial de la Infancia. La Infancia Amenazada, Nueva York, Fondo de las Naciones Unidas para la Infancia, 2005.

16. NACIONES UNIDAS: The Inequality Predicament. Report on The World Social Situation 2005, Nueva York, Naciones Unidas, D. of Economic and Social Affairs, 2005.

17. Con variaciones regionales: $80 \%$ en África occidental y central, Asia meridional, Oriente medio y África del norte. $28 \%$ en Asia oriental y Pacífico 
Violencias estructurales: obstáculos para el cumplimiento de los derechos humanos...

escolar asisten a la escuela secundaria. Un $27 \%$ asisten todavía a educación primaria (repiten curso o comenzaron tarde). La educación secundaria de las niñas es determinante para la igualdad entre género, más que con la enseñanza primaria, pues ayuda a las niñas a obtener mejores posiciones sociales ${ }^{18}$.

En la Unión Europea, como contexto general, destacan graves problemas entre los que se citan los siguientes: persistencia de niveles elevados de desempleo, en especial de larga duración; la baja tasa de empleo femenina; la desigual distribución de las tasas de edad en función de los grupos de más edad, pero también de los jóvenes; la persistencia de obstáculos para acceder al empleo de personas inmigrantes o pertenecientes a minorías étnicas y otros grupos desfavorecidos; las diferencias regionales en algunos Estados miembros que inciden en la cohesión social de Europa y la persistencia de problemas en los mercados de trabajo a nivel regional. La persistencia de la pobreza y la exclusión social, se reconocen como uno de los problemas más difíciles de resolver de la Unión Europea ya que hay un $9 \%$ de la población con pobreza persistente (pobres al menos durante dos de los últimos tres años). También se señalan como factores causales del desempleo los problemas de salud y la discapacidad, la separación familiar o la carencia de cualificaciones básicas. Se reconoce que el $15 \%$ de la población está en riesgo de caer en la pobreza ${ }^{19}$ y se estima que de no ser por la protección social recibida el riesgo de pobreza habría alcanzado el $24 \%$ (excluidas las pensiones) o el $40 \%$ (si se incluyen), con grandes diferencias entre estados miembros dependiendo de la cobertura e intensidad protectora de los respectivos sistemas, reconociéndose que la mayoría de los grupos de la población más afectados siguen siendo invisibles.

Hay grupos sociales mucho más expuestos a la pobreza en los que las mujeres están sobre-representadas, por ejemplo, las personas desempleadas, las familias monoparentales (principalmente las mujeres), las personas mayores que viven solas (principalmente mujeres) y las familias numerosas. Un caso especial de riesgo de pobreza y exclusion social es la de los jóvenes con dificultades y sin cualificación para acceder al mercado de trabajo. En 2002, un 19\% de las personas entre 18 y 24 años abandonaron el sistema escolar prematuramente y no siguieron ninguna formación ocupacional. En el caso de los menores, hay muchos de ellos en situación de vulnerabilidad con mayores tasas de pobreza de ingreso que los adultos (19\% en 2001), con el agravante de que las deprivaciones materiales en edades tempranas tienen graves consecuencias en todos los aspectos de su desarrollo y, por lo tanto, se reducen sus oportunidades futuras como adultos. Se estima que un $10 \%$ de la población menor de la UE está viviendo en hogares en los que ningún adulto tiene empleo ${ }^{20}$.

18. UNICEF: Progreso para la Infancia. Un balance entre la enseñanza primaria y la paridad entre los géneros. (2) Abril 2005 www.UNICEF.org

19. Con ingresos inferiores al $60 \%$ de los ingresos medios nacionales equivalentes.

20. COMISIÓN EUROPEA. Comunicación de la Comisión al Consejo, al Parlamento Europeo, al Comité Económico y Social Europeo y al Comité de las Regiones: Cuadro de Indicadores sobre la aplicación de la Agenda de Politica Social. COM (2003) 57 final. Bruselas 6.2.2003. Council of the 
La monoparentalidad, divorcio o separación son factores de pobreza o riesgo de ella. Según Olivier et. al. ${ }^{21}$ en la Unión Europea, una de cada cuatro familias monoparentales son pobres, proporción que aumenta en Alemania y Gran Bretaña y disminuye en Europa del sur por solidaridad familiar. El $84 \%$ de estos hogares están a cargo de mujeres (94\% en Portugal) siendo la mayoría mujeres divorciadas o separadas. Las prestaciones sociales representan el 34\% de los recursos. La tasa de actividad de las madres solas es del 68\% frente al $61 \%$ para las otras madres; el $90 \%$ en Suecia y entre $50-70 \%$ en los otros países, (en Gran Bretaña $51 \%$ frente al $61 \%$ de las otras madres). Hay diferencias entre estas familias, con un mayor riesgo a mayor número de hijos dependientes y dependiendo de diferentes modos de interactuar la precariedad social, la pobreza económica y la pobreza de condiciones de vida. Los hijos menores tienen mayor vulnerabilidad y riesgo de transmisión generacional de la pobreza.

Los dos Informes europeos realizados hasta el momento sobre la Igualdad entre mujeres y hombres ${ }^{22}$, destacan que se han logrado avances en los últimos años, pero reconocen que persisten importantes desigualdades por razón de género entre las que cabe destacar las siguientes:

- Aunque siguen reduciéndose las diferencias en educación (las jóvenes permanecen más tiempo en la educación o la formación profesional inicial) y empleo, persisten las diferencias salariales en contra de las mujeres y a estas les sigue resultando difícil compaginar sus responsabilidades familiares con un empleo a jornada completa, lo que les frena para acceder a un empleo que les permita sentirse seguras. La proporción media de empleo a tiempo parcial es de $30,4 \%$ en el caso de las mujeres y sólo un $6,6 \%$ en el de los hombres.

- La brecha salarial sigue siendo del $16 \%$ de media en la UE-15, y apenas ha cambiado en los últimos años. Considerando la UE-25 se sitúa en el 15\% ${ }^{23}$.

- Las diferencias entre las tasas de empleo femeninas y masculinas han disminuido un $0,5 \%$ hasta el $15,8 \%$ entre 2002 y 2003 , siendo la tasa de empleo femenino el $55,1 \%$. El grupo de edad con la tasa más baja es el de las mujeres jóvenes (14-25 años).

- Se incrementa el número de trabajadores pobres. No solo es necesario acceder a un empleo sino, también, que sea un empleo decente ${ }^{24}$.

European Union (Employment, Social Policy, Health and Consumer Affaires): Joint Report by the Commission and the Council in Social Inclusion, 4.3.2004.

21. OlIVIER, D. y EYDOUX, L. R.: Les familles monoparentales en Europe, 1ère partie y MARTIN, C. y MILLAR, J. $2^{\text {ème }}$ partie, Rennes, Université Rennes 2 - LAPSS - ENSP, (54) 2004.

22. COMISIÓN EUROPEA: Igualdad entre mujeres y hombres. 2004. Informe de la Comisión al Consejo, al Parlamento Europeo, al Comité Económico y Social Europeo y al Comité de las Regiones. Bruselas, 19.2.2004 COM(2004) 115 final. COMISIÓN EUROPEA: Igualdad entre mujeres y hombres 2005. Informe de la Comisión al Consejo, al Parlamento Europeo, al Comité Económico y Social Europeo y al Comité de las Regiones. Bruselas, 14.02.2005 COM (2005) 44 final.

23. EUROSTAT: Gender gaps in the reconciliation between work and family life. Statistics in Focus. Population and Social Conditions, 4/2005.

24. EUROSTAT: In-Work Poverty. Statistics in Focus. Population and Social Conditions, 5/2005. 
Violencias estructurales: obstáculos para el cumplimiento de los derechos humanos...

- En cuanto al desempleo, persisten las diferencias significativas con una tasa para las mujeres del 10\% frente a un 8,3\% de los hombres. Las mujeres siguen siendo más vulnerables que los hombres al desempleo y a la inactividad económica, sobre todo aquellas que tienen niveles educativos inferiores y las de menos edad o las de edad más avanzada. En los nuevos Estados miembros, las tasas de desempleo casi duplican las de la UE, si bien las diferencias en cuanto a desempleo tienden a ser menores.

- Disminuye aunque persiste la segregación por sexos del mercado de trabajo (17,5 \% segregación ocupacional y 25,2 \% segregación sectorial). Aunque aumentó el número de mujeres en profesiones liberales y de gestión de alto nivel como resultado de la mejora de sus cualificaciones, los hombres tienen el doble y el triple de probabilidades de ocupar puestos de gestión y dirección superior, estando también sub-representadas las mujeres en la mano de obra científica europea (30\%).

- Las mujeres siguen haciéndose cargo de la mayor parte del trabajo doméstico y familiar, lo que influye en su vida profesional y limita sus oportunidades de elección y promoción, sus salarios y sus pensiones. Los resultados de las medidas para la conciliación de la vida laboral y familiar se califican de bastante infructuosos, pues persiste la división tradicional de los cuidados y el trabajo retribuido entre las mujeres y los hombres. Las mujeres que tienen hijos pequeños tienen una tasa media de empleo $13,6 \%$ inferior a la de las mujeres sin hijos, asumen las tareas domésticas por lo que disponen de menos tiempo para el trabajo remunerado,

- No hay diferencias visibles entre mujeres y hombres en cuanto al riesgo de pobreza en general ${ }^{25}$, pero se señalan como grupos de riesgo de pobreza y exclusión social las mujeres de más edad y las que son cabezas de familia con personas dependientes a su cargo, estas últimas tienen el doble de riesgo de pobreza que la media de la UE (35\% en 2019). Las mujeres tienen menores derechos a la protección social cuando esta se basa en cotizaciones durante la vida laboral. Algunos países están adaptando sus sistemas con el reconocimiento de las actividades de cuidado de las mujeres a personas dependientes. Un nuevo grupo de riesgo que sufre una mayor situación de desventaja es el de las mujeres inmigrantes, con mayores tasas de empleos inseguros y peor remunerados (un 10\% menores que los de las mujeres no inmigrantes). La violencia doméstica aparece identificada como un riesgo de exclusión social para las mujeres en los Planes nacionales de acción para la inclusión social.

- Todos estos obstáculos y diferencias por razón de género se incrementan en los grupos de renta baja. Las mujeres pobres constituyen la mayoría de las personas económicamente inactivas por lo que son especialmente vulnerables para caer en la trampa de la pobreza ante rupturas familiares o violencia doméstica. También son más vulnerables al ser mayores o vivir solas con los

25. Cabe recordar lo comentado sobre las carencias metodológicas con perspectiva de género. 
hijos, el $35 \%$ de las familias monoparentales, encabezadas mayoritariamente por mujeres, viven en la pobreza.

Otro modo de acercarse al conocimiento de la situación de las mujeres europeas es en su calidad de miembros de familias ya que puede tener consecuencias tanto positivas como negativas. Como valoración subjetiva de la calidad de vida, la pertenencia a una familia se valora mucho por los ciudadanos de la UE-15 que la sitúan en tercer lugar, después salud e ingresos suficientes. Esta alta valoración se incrementa con los nuevos miembros EU-10- que la sitúan en segundo lugar, al mismo nivel que tener ingresos suficientes. Para estos últimos países, el factor más importante en la exclusión social percibida es el sentirse fuera de la familia, mientras que el ingreso es el menor y el desempleo no resulta estadísticamente significativo, parece que por ser una situación generalizada ${ }^{26}$.

Basado en la experiencia del trabajo diario, el análisis que realiza Cáritas Europa sobre la situación de las familias pobres en 44 países de Europa constata, en primer lugar, la existencia de una heterogeneidad de familias y de situaciones entre países, dependiendo de las diferencias en los sistemas de protección social. Se insiste en que la multidimensionalidad de la pobreza supone acercarse a ella desde una perspectiva de los derechos humanos fundamentales y teniendo en cuenta factores intangibles como vulnerabilidad, riesgo, desigualdad, marginación, discriminación, exclusión, sentimientos de impotencia, limitación de opciones y elecciones. Además, hay que tener en cuenta la heterogeneidad de situaciones posibles: pobreza temporal (intermitente) o crónica (círculo y reproducción generacional); leve, moderada o grave con falta crónica de recursos y degradación de las relaciones sociales agravada por un alto grado de dependencia externa. (trampa de la pobreza, circulo vicioso, transmisión intergeneracional). La pobreza supone estrés, riesgo de adicciones, delincuencia y violencias.

Cáritas considera como grupos de riesgo los siguientes: a) padres solos; b) familias numerosas; c) familias en las que uno o más miembros padecen enfermedad crónica, discapacidades, enfermedad mental, adicción a drogas o alcohol; d) retorno de personas desplazadas, refugiados, migrantes y solicitantes de asilo; e) personas desempleadas o con bajos ingresos. Hay una llamada de alerta hacía la situación de los menores con problemas de salud, dieta precaria, fracaso escolar y riesgo de pasar bajo protección pública a instituciones, así como el miedo de estas familias pobres a perder a sus hijos "que es su único bien». Nueve de cada diez padres solos son mujeres (son las que tienen más riesgo de pobreza, entre ellas, madres solteras) y las mujeres sufren las tasas más altas de desempleo, trabajan en economía sumergida, reciben salarios más bajos, su pre-

26. KrIEGER, H.: «Family Life» in Europe, Conference "Families, change and social policy in Europe». Dublin, 13-14 May 2004. 
Violencias estructurales: obstáculos para el cumplimiento de los derechos humanos...

cariedad laboral es mayor y su protección social menor o inexistente ${ }^{27}$. Análisis que comparte la Red Europea de Lucha contra la Pobreza ${ }^{28}$.

En cuanto a España, la realidad no difiere de la situación europea, con sus correspondientes diferencias territoriales, y este es el marco global de dificultades en el que hay que situar una gran parte de las desigualdades por razón de género que afectan a las mujeres pobres o en situación de exclusión social en edad activa pero que, como ya se ha señalado, tienen consecuencias en otras etapas de su ciclo vital, en niñas, jóvenes, adultas, mayores y muy mayores, además de repercutir en su ámbito familiar.

El Segundo Informe sobre la situación sociolaboral de la Mujer del Comité Económico y Social (CES, 2003), facilita una visión global y pormenorizada de nuestra realidad estructurada en cinco ámbitos: situación sociodemográfica; educación y formación; trabajo doméstico, responsabilidades familiares y usos del tiempo; participación social y política de las mujeres. Permite contextualizar el riesgo de pobreza humana que afecta a muchas mujeres, entendiendo no solo la pobreza de ingreso sino la falta de opciones de desarrollo humano en todos esos ámbitos. Considerando el empleo el principal factor de protección contra la pobreza y la exclusión social, encontramos que el desempleo español sigue siendo femenino (paro femenino ha sido el $63 \%$ del total); en el tiempo de permanencia en la situación de paro (factor clave en la empleabilidad) hay una diferencia negativa de más de diez puntos entre el porcentaje de mujeres que llevaban un año o más buscando empleo, en comparación a los varones ${ }^{29}$. Aunque el nivel de formación de las mujeres ha mejorado notablemente, la tasa de inserción es menor $y$, con frecuencia, el paro femenino se incrementa al aumentar el nivel de formación: de 1 a 1,4 en el nivel educativo más bajo; de 1 a 2,2 en la educación secundaria de segunda etapa y de 1 a 4,2 en la educación superior ${ }^{30}$. La tasa de paro más alta, tanto para hombres como para mujeres, corresponde al grupo de edad de 16 a 19 años, con bajos niveles de cualificación, alta temporalidad y alternancia empleo-paro (8,3\% varones y 7,4\% mujeres de ese grupo de edad) ${ }^{31}$. La alta incidencia de la temporalidad involuntaria de las mujeres conlleva consecuencias como menores salarios a igual cualificación y ocupación; menores oportunidades de formación y mayor riesgo de desempleo. Todas estas desventajas se concatenan y tienen su repercusión en la brecha salarial.

27. CÁRITAS EUROPA: Los rostros de la pobreza en Europa. La necesidad de politicas orientadas hacía la familia. $2^{\circ}$ Informe sobre la pobreza en Europa, Bruselas, febrero 2004.

28. MOSER, M. (EAPN): "Gender inequalities in social protection and social inclusión», en European Conference Women and Men in an Enlarged Europe, Malta 1-3 abril 2004.

29. Las tasas más bajas de inserción laboral desde la formación profesional corresponden a estudiantes de ambos sexos que eligieron profesiones más feminizadas y a la inversa.

30. Al concluir que una mejora del nivel formativo no es suficiente para las mujeres, el CES sugiere interrogarse sobre el tipo de formación recibida por las mujeres.

31. En educación, España es el segundo país europeo con mayores tasas de abandono escolar temprano $(29 \%)$. 
En cuanto a prestaciones económicas, a pesar de que el $60 \%$ de las personas registradas en los Servicios Públicos de Empleo fueron mujeres, el 54,6\% de los hombres se han beneficiado más de las prestaciones por desempleo del nivel contributivo que las mujeres $(45,4 \%)$. En cambio, en el nivel asistencial el porcentaje de mujeres fue de 2,2 puntos porcentuales mayor que el de los hombres, con sus negativas consecuencias al percibir cuantías económicas inferiores.

Los hogares monoparentales también tienen perfil femenino: un $7 \%$ del conjunto de las familias y nueve de cada diez están encabezados por mujeres cuyo perfil ha cambiado y se ha rejuvenecido, ya que se originan más en rupturas familiares que en la viudedad. La renta media anual de los hogares encabezados por mujeres es muy inferior a la de los hogares encabezados por hombres, por lo que se encuentran en mayor riesgo de pobreza.

Aunque la natalidad ha descendido, algo menos de la mitad de las mujeres en edad fértil manifiestan su deseo de ser madres con una media de hijos deseada de 2,1,lo que pone de manifiesto la existencia de obstáculos económicos, de salud y sociales a la maternidad ${ }^{32}$.

El trabajo doméstico y, como parte de él, la atención a lo miembros dependientes del hogar es otro factor de riesgo de pobreza humana y exclusión social para la mujeres, en cuanto suponen límites a sus condiciones de vida con obstáculos a la posibilidad de un empleo remunerado, salud, relaciones sociales, ocio y tiempo libre, etc. ${ }^{33}$ En el caso del empleo, la mayoría de las mujeres cuidadoras tienen como ocupación principal las tareas del hogar, aunque también las hay que tratan de compatibilizar los cuidados con un trabajo remunerado. En estos casos, hay muchas dificultades para conciliar vida laboral y familiar por lo que es fácil abandonar el empleo o trabajar a tiempo parcial, sin ser ello una elección libre. Estas mujeres cuidadoras tienen más problemas de salud que los hombres, no disponen de tiempo libre personal ni de vacaciones ( 24 horas los 365 días del año), considerando que las políticas públicas no reconocen su difícil situación al hacerlas responsables de un problema social ${ }^{34}$. El trabajo doméstico también es una fuente de ocupación para más de una cuarta parte de las mujeres inmigrantes $^{35}$, siendo frecuente la precariedad laboral y la economía sumergida lo que favorece míseros salarios y falta de protección social, con sus consecuencias a corto y medio plazo.

32. CES: Segundo Informe sobre la situación de las mujeres en la realidad sociolaboral española, Madrid, CES, 3/2003.

33. INE: Encuesta de empleo del tiempo. 2002/2003, Madrid, INE, 2003.

34. MARTíneZ ROMÁN, Ma A.: "Las familias ya no podemos más: Riesgos de exclusión social de las familias que cuidan a enfermos crónicos graves», Revista del Ministerio de Trabajo y Asuntos Sociales. Asuntos Sociales, 2002, pp. 145-165.

35. IMSERSO: Atención a las personas en situación de dependencia (Libro Blanco), Madrid, Ministerio de Trabajo y Asuntos Sociales-IMSERSO, 2005. 
Violencias estructurales: obstáculos para el cumplimiento de los derechos humanos...

España: Un contexto de Desiguales Oportunidades para las Mujeres

\begin{tabular}{|c|c|c|c|c|}
\hline $\begin{array}{l}\text { Situación socio- } \\
\text { demográfica }\end{array}$ & $\begin{array}{l}\text { Educación y } \\
\text { formación }\end{array}$ & $\begin{array}{c}\text { Empleo, si- } \\
\text { tuación socio- } \\
\text { profesional y } \\
\text { condiciones de } \\
\text { trabajo }\end{array}$ & $\begin{array}{c}\text { Trabajo domés- } \\
\text { tico, Responsa- } \\
\text { bilidades fami- } \\
\text { liares y usos del } \\
\text { tiempo }\end{array}$ & $\begin{array}{c}\text { Participación } \\
\text { social y política } \\
\text { de las mujeres }\end{array}$ \\
\hline $\begin{array}{l}\text { * Cambios en la } \\
\text { estructura de los } \\
\text { hogares: } \\
\text {. Disminución } \\
\text { tamaño medio. } \\
\text {. Feminización } \\
\text { monoparentales y } \\
\text { unipersonales } \\
\text { (menor renta). } \\
{ }^{*} \text { Cambios en la } \\
\text { composición y } \\
\text { dinámica pobla- } \\
\text { cional: } \\
\text {. Descenso de la } \\
\text { natalidad (1,25) } \\
\text {.Inmigración es } \\
\text { femenina. }\end{array}$ & $\begin{array}{l}\text { Avances } \\
\text { *Elevación del } \\
\text { nivel educativo: } \\
\text {. Incremento en } \\
\text { estudios universi- } \\
\text { tarios. } \\
\text {. Avance en la } \\
\text { formación con- } \\
\text { tinua. } \\
\text { *Mejor rendi- } \\
\text { miento educativo. } \\
\text { Desigualdades } \\
\text { *Segregación de } \\
\text { itinerarios forma- } \\
\text { tivos. } \\
\text { *Diferencias en } \\
\text { la transición de } \\
\text { la educación a } \\
\text { empleo. } \\
\text { Acceso más } \\
\text { tardío. } \\
\text {.Menor duración } \\
\text { del empleo. } \\
\text { *Menor inserción } \\
\text { laboral tras F.P. } \\
\text { ocupacional. }\end{array}$ & $\begin{array}{l}\text { *Crecimiento tasa } \\
\text { actividad. } \\
\text { PERO: } \\
\text { *La inactividad es } \\
\text { mayor } \\
\text { *El paro es feme- } \\
\text { nino: alta tasa y } \\
\text { persistente } \\
\text {. Es mayor la } \\
\text { permanencia en } \\
\text { paro:10 puntos } \\
\text { * Mayor el paro } \\
\text { mujeres jóvenes } \\
\text { *Nuevos perfiles } \\
\text { por estudiar } \\
\text { *Formación ne- } \\
\text { cesaria pero no } \\
\text { suficiente } \\
\text { *Mas asalariadas } \\
\text { y empleadoras } \\
\text { *Temporalidad } \\
\text { alta. } \\
\text { *Escaso empleo a } \\
\text { tiempo parcial } \\
\text { *Cambios en } \\
\text { diversificación } \\
\text { ocupaciones y } \\
\text { persistente no } \\
\text { cualificación } \\
\text {-Brecha salarial } \\
\text {-Mujeres inmi- } \\
\text { grantes subem- } \\
\text { pleo }\end{array}$ & $\begin{array}{l}\text { *Invisible la apor- } \\
\text { tación femenina } \\
\text { al sistema pro- } \\
\text { ductivo: } \\
\text {. Trabajo domés- } \\
\text { tico es femenino } \\
\text { (98\%) } \\
\text {. Doble, triple } \\
\text { carga por trabajo } \\
\text { fuera del hogar. } \\
\\
\text { * Como ocupa- } \\
\text { ción: Dificultades } \\
\text { de las condicio- } \\
\text { nes de trabajo } \\
\text { y bajos salarios. } \\
\text { *Carencias de } \\
\text { servicios } \\
\text { de apoyo a cuida- } \\
\text { dos suplidas por } \\
\text { FAMILIA. } \\
\text { *Aumento exce- } \\
\text { dencia cuidado } \\
\text { familiares y de } \\
\text { abandono em- } \\
\text { pleo. } \\
\text {. Escaso uso } \\
\text { permisos legales } \\
\text { los hombres } \\
\text { *Conciliación de } \\
\text { la vida familiar y } \\
\text { laboral como pro- } \\
\text { blema femenino }\end{array}$ & $\begin{array}{l}\text { *Baja represen- } \\
\text { tación } \\
\text { en todos los } \\
\text { ámbitos. }\end{array}$ \\
\hline
\end{tabular}

Fuente: Elaboración propia a partir de CES: Informe CES 3/2003. INE: Mujeres y Hombres en España, 2003. INE: Pobreza persistente en España 1994-2001. EUROSTAT: Gender gaps in the reconciliation between work and family life. Statistics in Focus. Population and Social Conditions, 4/2005. EUROSTAT: In-Work Poverty. Statistics in Focus. Population and Social Conditions, 5/2005.

Este panorama general de desigualdades que afectan a la mujer, es el telón de fondo para reconocer la violencia estructural sobre las condiciones de vida de las mujeres más pobres, incluyendo en el concepto de pobreza humana la pobreza económica que está muy ligada a la actividad ${ }^{36}$. Según el $\mathrm{INE}^{37}$, la mitad de los

36. Paro e inactividad son los mayores factores de riesgo de pobreza y ya se ha visto anteriormente cómo afecta a las mujeres en España.

37. INE: Mujeres y Hombres en España, Madrid, INE, 2003. 
pobres y pobres persistentes están inactivos, muchas de las mujeres pobres no pueden acceder a un empleo decente por estar obligadas a realizar actividades de cuidado no remuneradas ${ }^{38} \mathrm{y}$, además, eso las hace económicamente dependientes de la persona del hogar que puede obtener ingresos, lo que es un riesgo de violencia directa ${ }^{39}$. En el caso de poder realizar un actividad remunerada fuera del hogar, además de las actividades domésticas y de cuidado, su escasa o nula cualificación hace que estas mujeres pobres difícilmente accedan a un empleo decente según la OIT, encontrándose en un círculo vicioso del que no saben cómo salir.

La tasa de pobreza de las mujeres españolas es mucho mayor que la de los hombres, incluyendo la pobreza persistente; en el periodo 1994-2001 desciende dos puntos la tasa de pobreza de los hombres mientras que la de las mujeres se incrementa en algo más de medio punto; en el año 2001 del total de personas pobres las mujeres eran el $55,1 \%$, un $54 \%$ de los pobres permanentes y el $50,2 \%$ de los no pobres. Por edades, afecta más a los grupos de edad de 0-15 años (más de un quinto de los pobres) y más de 64 años y, en este último grupo, las mujeres tienen mayor riesgo de pobreza al envejecer, por la baja cuantía de sus pensiones relacionado con las altas tasas de desempleo, subempleos y economía sumergida, además del trabajo doméstico no remunerado y/o sin protección social.

Finalmente, una vez más desde la perspectiva de los derechos humanos y haciendo visible la violencia estructural, hay que hablar con las mujeres pobres ${ }^{40}$, reconociendo el derecho personal de cada una de ellas a un desarrollo humano para conocer lo qué piensan de sus condiciones de vida y cómo se sienten ante la acumulación de desventajas que sufren ${ }^{41}$. Aunque tienen elementos de discriminación en común, son muy heterogéneos las circunstancias de sus contextos y sus recursos, personales, familiares y sociales, hay que individualizar cada situación. Las mujeres entrevistadas nos han dicho que, como pobres, sienten vergüenza, dolor, con inseguridad permanente, impotencia, desesperanza, inutilidad social y pérdida total de autoestima. Este daño les resulta tan doloroso y difícil de soportar, que llega a causar depresión e ideas de suicidio y debería darse a conocer con el fin de romper con modalidades de violencia cultural que atribuyen la responsabilidad de la pobreza a las propias personas pobres, como si la pobreza fuera un estado libremente elegido, utilizando esta atribución para argumentar que las políticas sociales tienen sólo efectos perversos en cuanto

38. LA PARRA, D.: «Los cuidados de salud en las trayectorias biográficas», en Tortosa, J.M. (coord.): Mujeres pobres, indicadores de empobrecimiento en la España de hoy, Madrid, Fundación FOESSA, 2002, pp. 87-100.

39. ESPINAR, E.: "La violencia doméstica como factor de empobrecimiento", en Tortosa, J.M. (coord.): Mujeres pobres..., op.cit., pp. 101-125.

40. TORTOSA, J.M.: «El estudio sobre las mujeres y los estudios sobre pobreza: lo que queda por hacer», en Tortosa, J.M. (coord.): Mujeres pobres..., op.cit., pp. 153-165.

41. MARTíneZ ROMÁN, Ma A.: "Pistas para el diseño de políticas sociales: escuchando a las mujeres», en Tortosa, J.M. (coord.): Mujeres pobres..., op.cit., pp. 127-151. 
Violencias estructurales: obstáculos para el cumplimiento de los derechos humanos...

incentivan a las personas pobres a permanecer como beneficiarios dependientes de la ayuda social.

Las mujeres mayores perciben pensiones de muy baja cuantía y las mujeres en edad activa no pueden obtener ingresos suficientes, o no tienen trabajo o es un trabajo precario, mal remunerado, sin protección social. Estos empleos precarios son la causa de que pierdan la fuente de ingresos ante una enfermedad propia o de algunos de sus familiares (cuando exige que ellas les cuiden) y, la inseguridad laboral les mantiene en una situación continua de vulnerabilidad no sólo en el corto plazo sino, también, hipotecando su seguridad futura ya que, con razón, consideran que tienen una alta probabilidad de ser pobres en su vejez, por ello todas consideran que obtener un empleo «seguro» es la solución a sus problemas, quieren salir de su situación sin depender de nadie.

El sistema educativo y laboral está excluyendo, hay que reflexionar sobre la necesidad de adoptar políticas de prevención del fracaso escolar de las niñas y adolescentes y de prevención de los desajustes actuales en la transición de la escuela al mundo laboral. Además, en el caso de las mujeres que nos ocupan, es necesario tanto promover empleo para las mujeres como potenciar la mejora de su empleabilidad y esto es un proceso de larga duración que precisa muchos apoyos también de larga duración y una actuación integral psicosocial.

Y, en muchos casos, hay otros factores añadidos como la falta de viviendas sociales de alquiler que condiciona a las mujeres a vivir al día para obtener los ingresos que aseguren el pago de alquileres muy por encima de sus posibilidades, limitando sus posibilidades de mejorar sus expectativas al no poder hacer planes a medio o largo plazo. La falta de equipamiento de la vivienda también condiciona el que la mujer tenga que invertir mucho más tiempo y esfuerzo (generando stress) en conseguir los alimentos a bajo coste, conservarlos o lavar personalmente la ropa. A ello se añade el hecho de habitar en un barrio empobrecido y marginal, en cuanto supone un lastre para mejorar la empleabilidad porque residir en estos lugares no sólo supone una limitación de las posibilidades de encontrar empleo a través de las propias redes sociales sino que, además, genera un efecto de desconfianza y rechazo ante los posibles empleadores.

Todo ello se refuerza negativamente entre sí, acumulándose las dificultades y generando en las mujeres ese sentimiento de impotencia, de sentirse sin salida y, lo que es peor, de pérdida de su autoestima. Hay que flexibilizar las respuestas adaptándolas personalizadamente. Y hay que reconocer los derechos humanos a título individual, cuando las políticas sociales restringen los apoyos a la no existencia de familia, se está penalizando a las mujeres y a sus propias familias que desean ayudar. En el ámbito laboral, mejorar la empleabilidad de las mujeres también implica el reconocimiento de sus derechos humanos a título personal y no condicionarlas a la unidad de convivencia; acciones integradas en el marco de planes de igualdad de oportunidades abarcando los ámbitos de empleo, salud, educación, servicios sociales, protección social, vivienda..., teniendo en cuenta una política de desarrollo de un territorio específico que rompa con situaciones estructurales de desigualdad. Esto merece ser tenido en cuenta en las políticas, favoreciendo el encuentro y la organización de las mu- 
jeres y escuchando tanto la definición de sus necesidades como sus propuestas de solución.

Y, finalmente, hay que tener en cuenta algunas situaciones específicas que suponen un factor de vulnerabilidad añadido como es el caso de embarazos adolescentes, mujeres solas con cargas familiares, separaciones y divorcios, procesos migratorios, adicciones, minorías étnicas, (tanto de españolas como de extranjeras). Las políticas preventivas pueden evitar la vulnerabilidad de mujeres en estas condiciones y el coste, no solo social sino también económico, será mucho menor a la vez que garantizamos una eficiencia y eficacia de dichas políticas.

En conclusión, hay muchas mujeres en situación de pobreza humana en España y ello supone violencia estructural e injusticia social, porque es evitable. La sociedad debe conocer que las mujeres a lo largo de su ciclo vital (y sus hijos), se ven especialmente afectadas y discriminadas para poder superar su situación. Las políticas sociales no pueden esperar a actuar como última red de seguridad, en casos de pobreza extrema, sino que deben evitar que se generen estas situaciones mediante políticas preventivas de carácter integral que aseguren la igualdad de oportunidades de las mujeres, desde niñas, garantizando el reconocimiento efectivo de sus derechos humanos en sentido amplio, como condición necesaria para promover su pleno desarrollo humano e integración social. 